\title{
Steady State Analysis of the CLMS and Augmented CLMS Algorithms for Noncircular Complex Signals
}

\author{
Danilo P. Mandic ${ }^{1}$, Yili Xia ${ }^{1}$ and Scott C. Douglas ${ }^{2}$ \\ ${ }^{1}$ Imperial College London, London, UK, ${ }^{2}$ Southern Methodist University, Texas, USA \\ \{d.mandic, yili.xia06\}@imperial.ac.uk, douglas@engr.smu.edu
}

\begin{abstract}
The recently introduced augmented complex least mean square (ACLMS) algorithm is shown to be suitable for the processing of both second order circular (proper) and noncircular (improper) signals, by virtue of the underlying widely linear model. In theory, both the linear CLMS and widely linear ACLMS achieve the same mean square error for propers signals, whereas the ACLMS exhibits lower mean square error for improper signals. However, improperness can arise due to the system noise, input, or channel model and to shed more light on the convergence and steady state properties of ACLMS and CLMS in these cases we here employ the energy conservation principle. Simulations in adaptive prediction and system identification settings for signals with different probability distributions and degrees of noncircularity support the the analysis.
\end{abstract}

Index Terms-Widely linear modelling, augmented complex statistics, augmented CLMS (ACLMS), improperness, energy conservation principle, steady state analysis, convergence

\section{INTRODUCTION}

Complex-valued adaptive filtering algorithms have found numerous applications, such as noise cancellation, system identification and adaptive prediction [1], and are normally considered generic extensions of the corresponding real valued algorithms. For instance, consider a real-valued conditional mean estimator $y=E[y \mid x]$, which estimates the signal $\mathrm{y}$ based on the observation $x$. For zero mean, jointly normal $y$ and $x$, the optimal solution is the linear model, $\hat{y}=\mathbf{x}^{T} \mathbf{h}$, where $\mathbf{h}=\left[h_{1}, \ldots, h_{L}\right]^{T}$ is a vector of fixed filter coefficients, and $\mathbf{x}=\left[x_{1}, \ldots, x_{L}\right]^{T}$ is the regressor vector. In the complex domain, it is normally assumed that we can use the same form of estimator, leading to the standard complex linear minimum mean square error (MMSE) estimator

$$
\hat{y}=\mathbf{x}^{T} \mathbf{h}
$$

Note, however, that both the real and imaginary parts of complex variables are real, and hence

$$
\hat{y}_{r}=E\left[y_{r} \mid x_{r}, x_{i}\right], \quad \hat{y}_{i}=E\left[y_{i} \mid x_{r}, x_{i}\right]
$$

and a more general form of (1) can be expressed as

$$
\hat{y}=E\left[y_{r} \mid x_{r}, x_{i}\right]+\jmath E\left[y_{i} \mid x_{r}, x_{i}\right]
$$

Using $x_{r}=\left(x+x^{*}\right) / 2$ and $x_{i}=\left(x-x^{*}\right) / 2 \jmath$ we arrive at

$$
\hat{y}=E\left[y_{r} \mid x, x^{*}\right]+\jmath E\left[y_{i} \mid x, x^{*}\right]=E\left[y \mid x, x^{*}\right]
$$

leading to the widely linear estimator for complex valued data

$$
y=\mathbf{h}^{T} \mathbf{x}+\mathbf{g}^{T} \mathbf{x}^{*}=\mathbf{x}^{T} \mathbf{h}+\mathbf{x}^{H} \mathbf{g}
$$

where $\mathbf{h}$ and $\mathbf{g}$ are complex-valued coefficient vectors.
In practice, the widely linear estimate in (5) is produced by concatenating the input vector $\mathbf{x}$ with its conjugate $\mathbf{x}^{*}$, to give an augmented $2 L \times 1$ input vector $\mathbf{x}^{a}=\left[\mathbf{x}^{T}, \mathbf{x}^{H}\right]^{T}$, together with the augmented weights $\mathbf{w}^{a}=\left[\mathbf{h}^{T}, \mathbf{g}^{T}\right]^{T}$. The $2 L \times 2 L$ augmented covariance matrix [2]

$$
\mathbf{C}_{\mathbf{x x}}^{a}=E\left[\begin{array}{c}
\mathbf{x} \\
\mathbf{x}^{*}
\end{array}\right]\left[\mathbf{x}^{H} \mathbf{x}^{T}\right]=\left[\begin{array}{cc}
\mathbf{C}_{\mathbf{x x}} & \mathbf{P}_{\mathbf{x x}} \\
\mathbf{P}_{\mathbf{x x}}^{*} & \mathbf{C}_{\mathbf{x x}}^{*}
\end{array}\right]
$$

now contains the full second order information. From (6), it is clear that the covariance matrix, $\mathrm{C}_{\mathbf{x x}}=E\left[\mathbf{x x}^{H}\right]$, alone does not have sufficient degrees of freedom to describe full second order statistics [3], and in order to make use of all the available second order information we also need to consider the pseudocovariance matrix, $\mathrm{P}_{\mathbf{x x}}=E\left[\mathbf{x x}^{T}\right]$. Processes with the vanishing pseudo-covariance $\mathrm{P}_{\mathbf{x x}}=\mathbf{0}$ are termed second order circular (or proper), however, in most real world applications, complex signals are second order noncircular or improper.

Augmented complex statistics has opened the possibility to design adaptive filtering algorithms suitable for the processing of both proper and improper signals [2], [4]. These algorithms are usually termed "widely linear" or "augmented", such as the widely linear LMS (WL-LMS) [5], augmented CLMS (ACLMS), augmented affine projection (AAPA), and widely linear Recusive Least Squares (WL-RLS) algorithms in the adaptive filtering area [6], [7], [8], and also in the context of blind source separation [9], [10].

Widely linear adaptive filtering algorithms normally have superior performance over their conventional counterparts when dealing with noncircular complex signals, however, theoretical understanding of their performance is still subject of ongoing research [5], [11], [12]. In this paper, the analysis of steady state performance of CLMS and ACLMS is performed based on the conservation principle of the weight error energy flow throughout filter adaptation [1]. We cater for improperness of the teaching signal, input and noise, and evaluate steady state behavior of ACLMS and CLMS algorithms in such situations. In this paper, the following notations are adopted: $E[\cdot]$ denotes the statistical expectation of a vector or matrix, $\|\cdot\|$ the Euclidean norm of a vector, $(\cdot)^{*}$ the complex conjugate, $(\cdot)^{T}$ the transpose of a vector or a matrix, $(\cdot)^{H}$ the Hermitian transpose of a vector or a matrix, $\operatorname{Tr}(\cdot)$ the trace of a matrix, $\lambda_{\max }(\cdot)$ the largest eigenvalue of a matrix, $\otimes$ the Kronecker product operator, and $\mathbb{R}^{+}$the set of positive real numbers. 


\section{Steady STATE MEAN SQUARE PERFORMANCE OF CLMS AND ACLMS ON NONCIRCULAR SIGNALS}

Consider a second order noncircular (improper) teaching signal $d(k)$ described by the widely linear model

$$
d(k)=\mathbf{x}^{T}(k) \mathbf{h}_{\mathrm{o}}+\mathbf{x}^{H}(k) \mathbf{g}_{\mathrm{o}}+q(k)
$$

where $\mathbf{h}_{\mathrm{o}}$ and $\mathbf{g}_{\mathrm{o}}$ are respectively the optimal standard and conjugate weight vectors, $q(k)$ denotes the possibly improper measurement noise with variance $\sigma_{q}^{2}$, while $\mathbf{x}(k)$ is the complex-valued input vector $\mathbf{x}(k)=[x(k-1), \ldots, x(k-L)]^{T}$ with a positive-definite covariance matrix $\mathbf{C}_{\mathbf{x x}}=E\left[\mathbf{x} \mathbf{x}^{H}\right]$.

\section{A. Steady state analysis of CLMS for noncircular signals}

The conventional CLMS algorithm is given by [13]

$$
\begin{aligned}
y(k) & =\mathbf{x}^{T}(k) \mathbf{h}(k) \\
e(k) & =d(k)-y(k) \\
\mathbf{h}(k+1) & =\mathbf{h}(k)+\mu e(k) \mathbf{x}^{*}(k)
\end{aligned}
$$

where $\mathbf{h}(k)$ is the $L \times 1$ weight vector at time instant $k, y(k)$ the output signal, $e(k)$ output error, and $\mu$ the step size. The steady state performance is quantified by the mean square error (MSE) given by

$$
\mathrm{MSE}=\lim _{k \rightarrow \infty} E\left[|e(k)|^{2}\right]
$$

To find a closed form of MSE of CLMS for noncircular signals (generated by the widely linear signal model in (7)), we shall first define the weight error vector $\tilde{\mathbf{h}}(k)=\mathbf{h}_{\mathrm{o}}-\mathbf{h}(k)$; then upon subtracting the weight update in (8) from $\mathbf{h}_{\mathrm{o}}$, we have

$$
\tilde{\mathbf{h}}(k+1)=\tilde{\mathbf{h}}(k)-\mu \mathbf{x}^{*}(k) \mathbf{e}(k)
$$

Pre-multiplying both sides with $\mathbf{x}^{T}(k)$ gives

$$
\mathbf{x}^{T}(k) \tilde{\mathbf{h}}(k+1)=\mathbf{x}^{T}(k) \tilde{\mathbf{h}}(k)-\mu\|\mathbf{x}\|^{2} e(k)
$$

allowing us to introduce the a posteriori and a priori errors $e_{p}(k)$ and $e_{a}(k)$ as [1]

$$
e_{p}(k)=\mathbf{x}^{T}(k) \tilde{\mathbf{h}}(k+1) \text { and } e_{a}(k)=\mathbf{x}^{T}(k) \tilde{\mathbf{h}}(k)
$$

and to rewrite the output error $e(k)$ as

$$
e(k)=d(k)-y(k)=e_{a}(k)+\mathbf{x}^{H} \mathbf{g}_{o}+q(k)
$$

Since $\mathrm{MSE}=\mathrm{EMSE}+J_{\min }$ where the excess mean square error (EMSE) arising due to the mismatch between the filter weights and optimal weights is defined as

$$
\mathrm{EMSE}=\lim _{k \rightarrow \infty} E\left[\left|e_{a}(k)\right|^{2}\right]
$$

this illustrates that the mimimum achievable MSE for CLMS operating on improper signals

$$
J_{\min }>\sigma_{q}^{2}
$$

as opposed to $J_{\min }=\sigma_{q}^{2}$ for circular signals. Compared with the standard analysis of CLMS in [1], the additional term $\mathbf{x}^{H} \mathbf{g}_{o}$ arises due to the improper nature of the desired signal in (7). The a posteriori and a priori errors in (12) are related by

$$
e_{p}(k)=e_{a}(k)-\mu\|\mathbf{x}(k)\|^{2} e(k)
$$

and for a nonzero input $\mathbf{x}(k)$, we have

$$
\mu e(k)=\frac{e_{p}(k)-e_{a}(k)}{\|\mathbf{x}\|^{2}}
$$

The evolution of the weight error vector $\tilde{\mathbf{h}}(k)$ in (11) can now be expressed as

$$
\tilde{\mathbf{h}}(k+1)=\tilde{\mathbf{h}}(k)-\frac{e_{p}(k)-e_{a}(k)}{\|\mathbf{x}\|^{2}} \mathbf{x}^{*}(k)
$$

or equivalently

$$
\tilde{\mathbf{h}}(k+1)+\frac{\mathbf{x}^{*}(k)}{\|\mathbf{x}(k)\|^{2}} e_{a}(k)=\tilde{\mathbf{h}}(k)+\frac{\mathbf{x}^{*}(k)}{\|\mathbf{x}(k)\|^{2}} e_{p}(k)
$$

Square both sides to obtain the following 'energy conservation' relation describing the evolution of the weight error vector

$$
\|\tilde{\mathbf{h}}(k+1)\|^{2}+\frac{\left|e_{a}(k)\right|^{2}}{\|\mathbf{x}(k)\|^{2}}=\|\tilde{\mathbf{h}}(k)\|^{2}+\frac{\left|e_{p}(k)\right|^{2}}{\|\mathbf{x}(k)\|^{2}}
$$

If we apply the statistical expectation of both sides of (20), and assume that at the steady state, $E\left[\|\tilde{\mathbf{h}}(k+1)\|^{2}\right] \simeq$ $\| E\left[\tilde{\mathbf{h}}(k) \|^{2}\right]$, then

$$
E\left[\frac{\left|e_{a}(k)\right|^{2}}{\|\mathbf{x}(k)\|^{2}}\right]=E\left[\frac{\left|e_{p}(k)\right|^{2}}{\|\mathbf{x}(k)\|^{2}}\right]
$$

Substituting (20) into the RHS of (21), gives

$$
E\left[\frac{\left|e_{a}(k)\right|^{2}}{\|\mathbf{x}(k)\|^{2}}\right]=E\left[\frac{\left|e_{a}(k)-\mu\|\mathbf{x}(k)\|^{2} e(k)\right|^{2}}{\|\mathbf{x}(k)\|^{2}}\right]
$$

and upon combining with (13), this yields

$E\left[\frac{\left|e_{a}(k)\right|^{2}-\left|e_{a}(k)-\mu\|\mathrm{x}(k)\|^{2}\left(e_{a}(k)+q(k)+\mathbf{x}^{H} \mathbf{g}_{o}\right)\right|^{2}}{\|\mathbf{x}(k)\|^{2}}\right]=0$

We can now use the standard assumptions that at the steady state (as $k \rightarrow \infty$ ), the noise sequence $q(k)$ is i.i.d. and statistically independent of the input sequence $\mathbf{x}(k)$, and that $e_{a}(k)$ is independent of $\mathbf{x}(k)$, to obtain

$$
\begin{aligned}
2 \mathrm{EMSE}_{\mathrm{CLMS}} & =\mu E\left[\|\mathbf{x}(k)\|^{2}\left|e_{a}(k)\right|^{2}\right] \\
& +\mu E\left[\|\mathbf{x}(k)\|^{2}\left|\mathbf{x}^{H} \mathbf{g}_{o}\right|^{2}\right]+\mu \sigma_{q}^{2} \operatorname{Tr}\left(\mathbf{C}_{\mathbf{x x}}\right)
\end{aligned}
$$

For a sufficiently small step size $\mu$, we can neglect the term $\mu E\left[\|\mathbf{x}(k)\|^{2}\left|e_{a}(k)\right|^{2}\right]$ to give

$$
\operatorname{EMSE}_{\mathrm{CLMS}}=\frac{\mu}{2} \sigma_{q}^{2} \operatorname{Tr}\left(\mathbf{C}_{\mathbf{x x}}\right)+\frac{\mu}{2} E\left[\|\mathbf{x}(k)\|^{2}\left|\mathbf{x}^{H} \mathbf{g}_{o}\right|^{2}\right]
$$

For a large value of the step size $\mu$, the second term on the RHS of (24) cannot be neglected; with a further assumption that at the steady state $\|\mathbf{x}(k)\|^{2}$ is statistically independent of $\left|e_{a}(k)\right|^{2}$, we have

$$
\mathrm{EMSE}_{\mathrm{CLMS}}=\frac{\mu \sigma_{q}^{2} \operatorname{Tr}\left(\mathbf{C}_{\mathbf{x x}}\right)+\mu E\left[\|\mathbf{x}(k)\|^{2}\left|\mathbf{x}^{H} \mathbf{g}_{o}\right|^{2}\right]}{2-\operatorname{Tr}\left(\mathbf{C}_{\mathbf{x x}}\right)}
$$

Compared with the expressions for EMSE for standard CLMS operating on proper signals generated by a strictly linear model (see [14], [1]), both (24) and (25) contain an additional "conjugate" term $\mathbf{x}^{H}(k) \mathbf{g}_{o}$, arising from the widely linear nature of the teaching signal model in (7), thus illustrating that the standard CLMS is suboptimal for the filtering of second order noncircular signals (from (7), ideally $J_{\min }=\sigma_{q}^{2}$ ). 
Using (13), we finally obtain the steady state mean square error of standard CLMS for improper signals in the form

$$
\mathrm{MSE}_{\mathrm{CLMS}}=\mathrm{EMSE}_{\mathrm{CLMS}}+E\left[\left|\mathbf{x}^{H} \mathbf{g}_{o}\right|^{2}\right]+\sigma_{q}^{2}
$$

where due to the inadequacy of the strictly linear CLMS to estimate improper signals produced by the widely model in (7), the minimum MSE of CLMS, $J_{\min }=E\left[\left|\mathbf{x}^{H} \mathbf{g}_{o}\right|^{2}\right]+\sigma_{q}^{2}$ is larger than the optimal one $J_{\min }=\sigma_{q}^{2}$; this mismatch depends on the degree of noncircularity reflected by the size of the conjugate weight vector $\mathbf{g}_{o}$.

\section{B. Steady state analysis of ACLMS for noncircular signals}

The widely linear version of CLMS, called the augmented CLMS (ACLMS), was proposed in [6], [14] and can be summarised as

$$
\begin{aligned}
y(k) & =\mathbf{x}^{T}(k) \mathbf{h}(k)+\mathbf{x}^{H}(k) \mathbf{g}(k) \\
e(k) & =d(k)-y(k) \\
\mathbf{h}(k+1) & =\mathbf{h}(k)+\mu e(k) \mathbf{x}^{*}(k) \\
\mathbf{g}(k+1) & =\mathbf{g}(k)+\mu e(k) \mathbf{x}(k)
\end{aligned}
$$

where $\mathbf{h}(k)$ and $\mathbf{g}(k)$ are respectively the standard and conjugate weight vectors. Similarly to the analysis in the previous subsection, by subtracting the $\mathbf{h}$ and $\mathbf{g}$ weight updates in (27) from their respective optimal weights $\mathbf{h}_{\mathrm{o}}$ and $\mathbf{g}_{\mathrm{o}}$, we obtain the corresponding weight error vectors $\tilde{\mathbf{h}}(k)=\mathbf{h}_{\mathrm{o}}-\mathbf{h}(k)$ and $\tilde{\mathbf{g}}(k)=\mathbf{g}_{0}-\mathbf{g}(k)$, whose evolution is described by

$$
\begin{aligned}
\tilde{\mathbf{h}}(k+1) & =\tilde{\mathbf{h}}(k)-\mu \mathbf{x}^{*}(k) e(k) \\
\tilde{\mathbf{g}}(k+1) & =\tilde{\mathbf{g}}(k)-\mu \mathbf{x}(k) e(k)
\end{aligned}
$$

Multiply both sides of (28) with $\mathbf{x}^{T}(k)$ and both sides of (29) with $\mathbf{x}^{H}(k)$ to yield

$$
\begin{aligned}
\mathbf{x}^{T}(k) \tilde{\mathbf{h}}(k+1) & =\mathbf{x}^{T}(k) \tilde{\mathbf{h}}(k)-\mu\|\mathbf{x}\|^{2} e(k) \\
\mathbf{x}^{H}(k) \tilde{\mathbf{g}}(k+1) & =\mathbf{x}^{H}(k) \tilde{\mathbf{g}}(k)-\mu\|\mathbf{x}\|^{2} e(k)
\end{aligned}
$$

The a posteriori and a priori errors $e_{p}(k)$ and $e_{a}(k)$ now become

$$
\begin{aligned}
& e_{p}(k)=\mathbf{x}^{T}(k) \tilde{\mathbf{h}}(k+1)+\mathbf{x}^{H}(k) \tilde{\mathbf{g}}(k+1) \\
& e_{a}(k)=\mathbf{x}^{T}(k) \tilde{\mathbf{h}}(k)+\mathbf{x}^{H}(k) \tilde{\mathbf{g}}(k)
\end{aligned}
$$

with

$$
e_{p}(k)=e_{a}(k)-2 \mu\|\mathbf{x}\|^{2} e(k)
$$

and

$$
e(k)=\frac{1}{2 \mu\|\mathbf{x}\|^{2}}\left(e_{p}(k)-e_{a}(k)\right)
$$

Processing in the same manner as for CLMS, with a further assumption that at the steady state $\tilde{\mathbf{h}}(k+1) \simeq \tilde{\mathbf{h}}(k)$ and $\tilde{\mathbf{g}}(k+$ $1) \simeq \tilde{\mathbf{g}}(k)$, we have

$$
\begin{array}{r}
\|\tilde{\mathbf{h}}(k+1)\|^{2}+\frac{\tilde{\mathbf{h}}^{H}(k) \mathbf{x}^{*}(k) e_{a}(k)}{2\|\mathbf{x}\|^{2}}= \\
\|\tilde{\mathbf{h}}(k)\|^{2}+\frac{\tilde{\mathbf{h}}^{H}(k+1) \mathbf{x}^{*}(k) e_{p}(k)}{2\|\mathbf{x}\|^{2}}
\end{array}
$$

and

$$
\begin{array}{r}
\|\tilde{\mathbf{g}}(k+1)\|^{2}+\frac{\tilde{\mathbf{g}}^{H}(k) \mathbf{x}^{*}(k) e_{a}(k)}{2\|\mathbf{x}(k)\|^{2}}= \\
\|\tilde{\mathbf{g}}(k)\|^{2}+\frac{\tilde{\mathbf{g}}^{H}(k+1) \mathbf{x}^{*}(k) e_{p}(k)}{2\|\mathbf{x}(k)\|^{2}}
\end{array}
$$

Adding up (35) and (36) gives the weight error energy conservation equations

$$
\begin{array}{r}
\|\tilde{\mathbf{h}}(k+1)\|^{2}+\|\tilde{\mathbf{g}}(k+1)\|^{2}+\frac{\left|e_{a}(k)\right|^{2}}{2\|\mathbf{x}(k)\|^{2}}= \\
\|\tilde{\mathbf{h}}(k)\|^{2}+\|\tilde{\mathbf{g}}(k)\|^{2}+\frac{\left|e_{p}(k)\right|^{2}}{2\|\mathbf{x}(k)\|^{2}}
\end{array}
$$

Taking the statistical expectation, and assuming that at the steady state $E\left[\|\tilde{\mathbf{h}}(k+1)\|^{2}\right] \simeq \| E\left[\tilde{\mathbf{h}}(k) \|^{2}\right]$, gives

$$
E\left[\frac{\left|e_{a}(k)\right|^{2}}{\|\mathbf{x}(k)\|^{2}}\right]=E\left[\frac{\left|e_{p}(k)\right|^{2}}{\|\mathbf{x}(k)\|^{2}}\right]
$$

Similar to the analysis in Section II-A, it follows immediately that

$$
2 \mathrm{EMSE}_{\mathrm{ACLMS}}=\mu E\left[\|\mathbf{x}(k)\|^{2}\left|e_{a}(k)\right|^{2}\right]+\mu \sigma_{q}^{2} \operatorname{Tr}\left(\mathbf{C}_{\mathbf{x x}}\right)
$$

For a sufficiently small step size $\mu$, we obtain

$$
\mathrm{EMSE}_{\mathrm{ACLMS}}=\frac{\mu}{2} \sigma_{q}^{2} \operatorname{Tr}\left(\mathbf{C}_{\mathbf{x x}}\right)
$$

whereas for a large value of $\mu$, we have

$$
\operatorname{EMSE}_{\mathrm{ACLMS}}=\frac{\mu \sigma_{q}^{2} \operatorname{Tr}\left(\mathbf{C}_{\mathbf{x x}}\right)}{2-\operatorname{Tr}\left(\mathbf{C}_{\mathbf{x x}}\right)}
$$

Note that from

$$
\begin{aligned}
e(k) & =d(k)-y(k) \\
& =\mathbf{x}^{T}(k)\left(\mathbf{h}_{o}(k)-\tilde{\mathbf{h}}(k)\right)+\mathbf{x}^{H}(k)\left(\mathbf{g}_{o}(k)-\tilde{\mathbf{h}}(k)\right)+q(k) \\
& =e_{a}(k)+q(k)
\end{aligned}
$$

the mean square error of ACLMS, denoted by MSE $\mathrm{ACLMS}_{\text {, has }}$ the form

$$
\mathrm{MSE}_{\mathrm{ACLMS}}=\mathrm{EMSE}_{\mathrm{ACLMS}}+\sigma_{q}^{2}
$$

As desired, the minimum achievable mean square error $J_{\min }=$ $\sigma_{q}^{2}$, giving theoretical justification for the performance advantage of ACLMS over CLMS when dealing with second order noncircular signals; this conforms with the result in [12] showing that the minimum MSE of CLMS is larger than that of ACLMS for noncircular signals.

\section{MEAN AND MEAN-SQUARE STABILITY ANALYSIS OF ACLMS}

We shall next investigate the bounds on the step size $\mu$, giving insight into the convergence of the ACLMS algorithm. From Section II-B, the vector-matrix form for the weight error vectors becomes

$$
\begin{aligned}
{\left[\begin{array}{c}
\tilde{\mathbf{h}}(k+1) \\
\tilde{\mathbf{g}}(k+1)
\end{array}\right] } & =\left[\begin{array}{cc}
\mathbf{I}-\mu \mathbf{x}^{*}(k) \mathbf{x}^{T}(k) & -\mu \mathbf{x}^{*}(k) \mathbf{x}^{H}(k) \\
-\mu \mathbf{x}(k) \mathbf{x}^{T}(k) & \mathbf{I}-\mu \mathbf{x}(k) \mathbf{x}^{H}(k)
\end{array}\right]\left[\begin{array}{c}
\tilde{\mathbf{h}}(k) \\
\tilde{\mathbf{g}}(k)
\end{array}\right] \\
& +\mu\left[\begin{array}{c}
\mathbf{x}^{*}(k) \\
\mathbf{x}(k)
\end{array}\right] q(k)
\end{aligned}
$$


TABLE I

STEP SIZE BOUNDS OF ACLMS FOR THE IMPROPER PROCESS IN (50) DRIVEN BY DOUBLY WHITE CIRCULAR GAUSSIAN AND UNIFORM DRIVING NOISES

\begin{tabular}{|c|c|c|c|c|}
\hline Types of input & $\frac{2}{\lambda_{\max }\left(\mathcal{C}_{\mathrm{xx}}^{a}\right)}$ & $\frac{1}{\lambda_{\max }\left(A^{-1} B\right)}$ & $\frac{1}{\max \left(\lambda(H) \in \mathbb{R}^{+}\right)}$ & $\mu_{\max }$ \\
\hline Gaussian input & 1.2795 & 1.2853 & 0.9109 & 0.9109 \\
\hline Uniform input & 0.9769 & 0.9752 & 0.6614 & 0.6614 \\
\hline
\end{tabular}

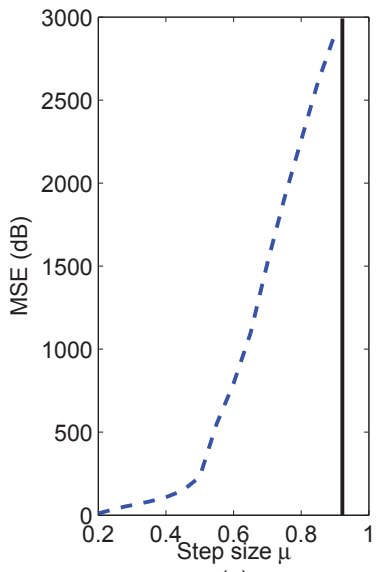

(a)

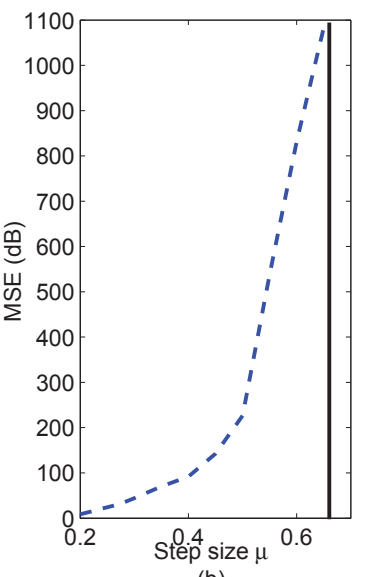

(b)

Fig. 1. Simulated MSE (dashed line) and theoretical bounds (solid line) of ACLMS as a function of step-size $\mu$ for (a) Gaussian input (b) Uniform input.

Upon applying the statistical expectation operator, and using the 'augmented' notation for the weight error vector $\tilde{\mathbf{w}}(k)=$ $\left[\tilde{\mathbf{h}}^{T}(k), \tilde{\mathbf{g}}^{T}(k)\right]^{T}$, we obtain

$$
E[\tilde{\mathbf{w}}(k+1)]=\left(\mathbf{I}-\mu \mathbf{C}_{\mathbf{x x}}^{a *}\right) E[\tilde{\mathbf{w}}(k)]
$$

Performing the analysis of modes of convergence with respect to the conjugate augmented covariance matrix $\mathbf{C}_{\mathbf{x x}}^{a *}$ as in [11], and bearing in mind that the eigenvalues of $\mathbf{C}_{\mathbf{x x}}^{a *}$ are identical to those of $\mathbf{C}_{\mathbf{x x}}^{a}$, it is easy to show that the step size is bounded by

$$
0<\mu<\frac{2}{\lambda_{\max }\left(\mathbf{C}_{\mathbf{x x}}^{a}\right)}
$$

where $\lambda_{\max }\left(\mathbf{C}_{\mathbf{x x}}^{a}\right)$ is the largest eigenvalue of the augmented covariance matrix $\mathbf{C}_{\mathbf{x x}}^{a}$.

Noting that the expression (45) satisfies the form of the evolution of the mean weight error vector, then, as shown in [1], the bounds on the step size $\mu$ can be made tighter based on $\lambda(F(\mu))<1$, where

$$
F(\mu)=\mathbf{I}-\mu A+\mu^{2} B
$$

and $A=E\left[P^{T}(k)\right] \otimes \mathbf{I}+\mathbf{I} \otimes E\left[P^{T}(k)\right], B=E\left[P^{T}(k)\right] \otimes$ $E[P(k)]$, and $P(k)=\mathbf{x}^{a}(k) \mathbf{x}^{a H}(k)$. Following the argument used in [15], we can finally establish the bound on the stepsize in the form

$$
0<\mu<\min \left\{1 / \lambda_{\max }\left(A^{-1} B\right), 1 / \max \left(\lambda(H) \in \mathbb{R}^{+}\right)\right\}
$$

where $H$ is defined by

$$
H=\left[\begin{array}{cc}
(1 / 2) A & -(1 / 2) B \\
\mathbf{I} & \mathbf{0}
\end{array}\right]
$$

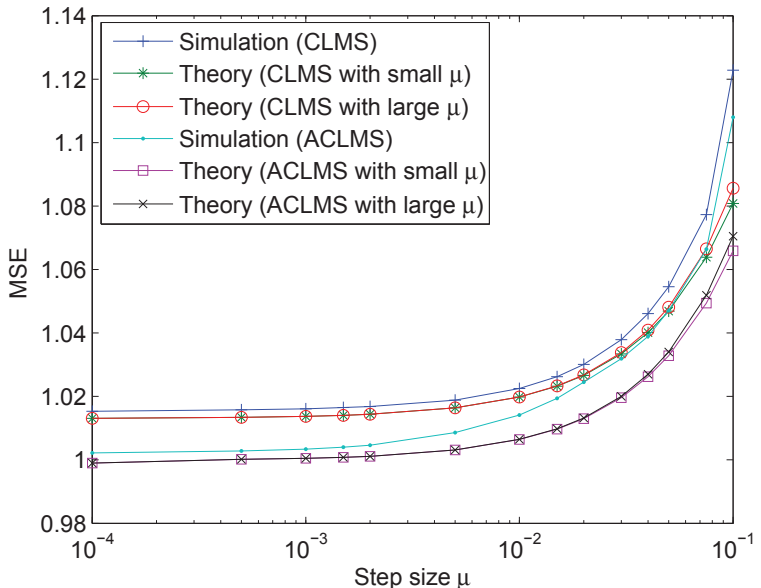

(a) Gaussian input

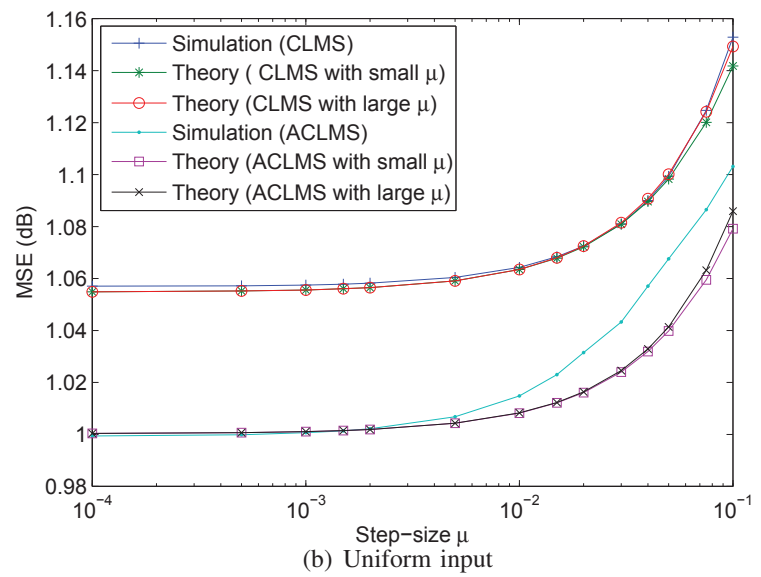

Fig. 2. Comparison of theoretical and simulated steady state MSE of CLMS and ACLMS for noncircular AR signals generated by (a) Gaussian driving noise and (b) Uniform driving noise.

\section{Simulations}

The first set of simulations was performed in an one step prediction setting, for a noncircular complex signal generated by the widely linear autoregressive (AR) model

$$
r(k+1)=0.5 r(k)+0.2 r^{*}(k)+q(k)
$$

All the graphs were produced by averaging 200 independent trials. Our analysis does not require the input data to be Gaussian, and two cases of complex valued doubly white circular driving noise $q(k)$ were investigated: Gaussian and uniform, both with zero mean and unit variance. Table I presents the theoretical bounds on the step size $\mu$ of the ACLMS algorithm; this is further illustrated in Fig. 1 which 

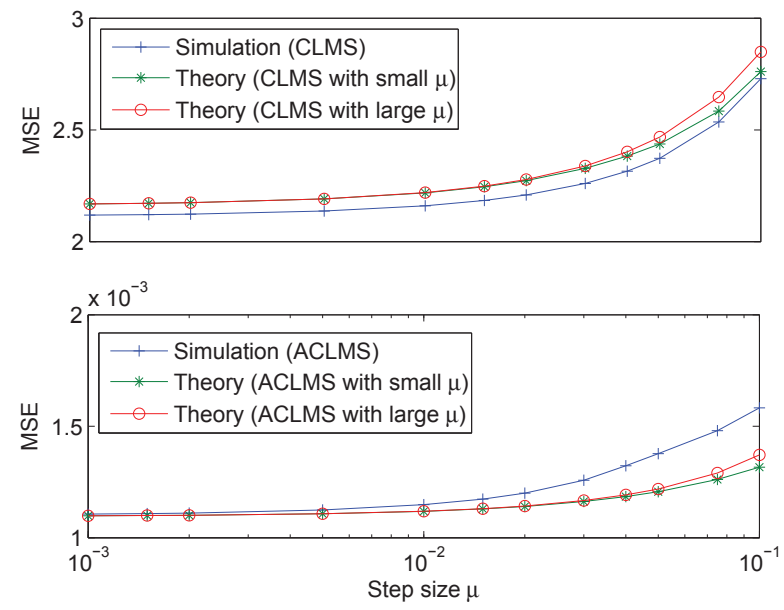

(a) Results for $\rho=0.1$
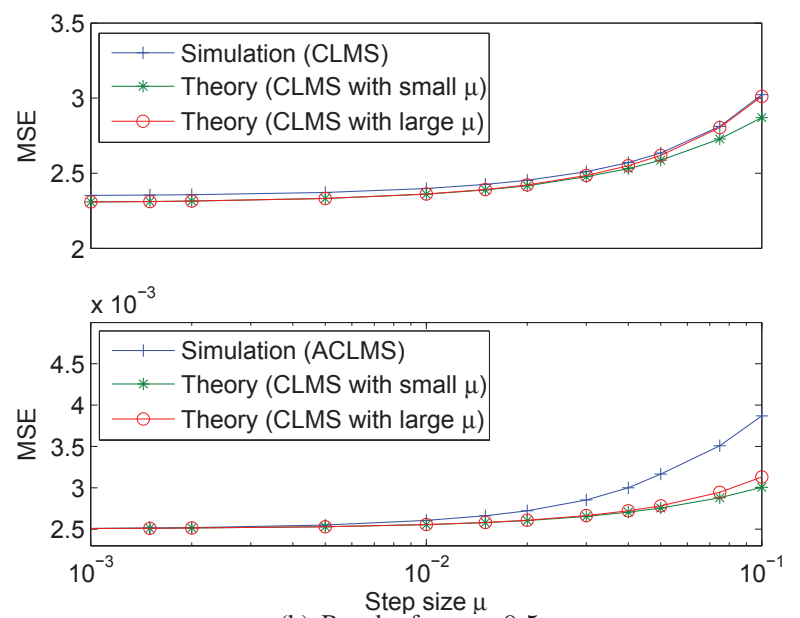

(b) Results for $\rho=0.5$

Fig. 3. Comparison of the theoretical and simulated steady state MSE of CLMS and ACLMS algorithms: (a) noncircular noise $(\rho=0.1)$, and (b) nonciruclar noise $(\rho=0.5)$.

shows both the simulated MSE curves (dashed line) and theoretical bounds (solid line). Fig. 2 shows the simulated and theoretical steady state MSE curves of CLMS and ACLMS as a function of step size $\mu$ - they are in good agreement with the theoretical results, estimated using (26) and (43).

The next set of simulations was performed in a system identification setting, where the system to be identified was a strictly linear FIR channel of length $L=4$ with coefficients $\mathbf{h}_{o}=[1.79,-1.85,1.27,-0.41]^{T}$; the input signal $x(k)$ was circular complex valued doubly white Gaussian noise with zero mean and unit variance. Following the discussion in [4], the desired signal was real valued $d(k)=\operatorname{Re}\left\{\mathbf{x}^{T}(k) \mathbf{h}_{o}\right\}$, and the output of the filter was contaminated by noncircular Gaussian noise

$$
q(k)=\sqrt{1-\rho^{2}} q_{r}(k)+\jmath \rho q_{i}(k)
$$

at an SNR level of $30 \mathrm{~dB}$; the components $q_{r}(k)$ and $q_{i}(k)$ are uncorrelated real valued white Gaussian processes with zero mean and unit variance (double whiteness). By changing the value of $\rho \in[0,1]$, we can control the degree of noncircularity of $q(k)$. Fig. 3 illustrates the theoretical and simulated MSE of the CLMS and ACLMS algorithms for two cases of noncircular noise $(\rho=0.1,0.5)$. In both the cases, theoretical results were in good agreement with the simulations, and as desired the ACLMS algorithm provided smaller MSE.

\section{CONCLUSION}

The mean square performance analysis of the standard complex least mean square (CLMS) and the widely linear augmened CLMS (ACLMS) algorithm has been performed for the filtering of second order noncircular complex signals. It has been shown that CLMS is suboptimal for second order noncircular processes, and the advantage of ACLMS over CLMS has been quantified using the energy conservation approach. Simulations in the widely linear prediction and system identification scenarios support the analysis.

\section{REFERENCES}

[1] A. H. Sayed, Adaptive Filters, John Wiley \& Sons, 2008.

[2] P. J. Schreier and L. L. Scharf, "Second-order analysis of improper complex random vectors and process," IEEE Trans. Signal Process., vol. 51, no. 3, pp. 714-725, 2003.

[3] F. D. Neeser and J. L. Massey, "Proper complex random processes with applications to information theory," IEEE Trans. Inform. Theory, vol. 39, no. 4, pp. 1293-1302, 1993.

[4] B. Picinbono and P. Chevalier, "Widely linear estimation with complex data," IEEE Trans. Signal Process., vol. 43, no. 8, pp. 2030-2033, 1995.

[5] R. Schober, W. H. Gerstacker, and L. H. J. Lampe, "Date-aided and blind stochastic gradients for widely linear MMSE MAI supression for DS-CDMA," IEEE Trans. Signal Process., vol. 52, no. 3, pp. 746-756, 2004.

[6] S. Javidi, S. L. Goh M. Pedzisz, and D. P. Mandic, "The augmented complex least mean square algorithm with application to adaptive prediction problems," Proc. Ist IARP Workshop Cogn. Inform. Process., pp. 54-57, 2008.

[7] S. C. Douglas, "Widely linear Recursive Least-Squares algorithm for adaptive beamforming," Proc. IEEE Int. Conf. Acoust., Speech Signal Process., pp. 2041-2044, 2009.

[8] Y. Xia, C. Cheong-Took, and D. P. Mandic, "An augmented affine projection algorithm for the filtering of noncircular complex signals," Signal Process., vol. 90, no. 6, pp. 1788-1799, 2010.

[9] S. C. Douglas, "Fixed-point algorithms for the blind separation of arbitrary complex-valued non-Gaussian signal mixtures," EURASIP $J$. Adv. Signal Process., vol. 2007, no. 1, pp. 83-83, 2007.

[10] S. Javidi, D. P. Mandic, and A. Cichocki, "Complex blind source extraction from noisy mixtures using second-order statistics," IEEE Trans. Circuits Syst. I, vol. 57, no. 7, pp. 1404-1416, 2010.

[11] S. C. Douglas and D. P. Mandic, "Mean and mean-square analysis of the complex LMS algorithm for non-circular Gaussian signals," Proc. IEEE Workshop Digital Signal Process., pp. 101-106, 2009.

[12] S. C. Douglas and D. P. Mandic, "Performance analysis of the conventional complex LMS and augmented complex LMS algorithms," Proc. IEEE Int. Conf. Acoust., Speech Signal Process., pp. 3794-3797, 2010.

[13] B. Widrow, J. McCool, and M. Ball, “The complex LMS algorithm," Proc. IEEE, vol. 63, pp. 719-720, 1975.

[14] D. P. Mandic and S. L. Goh, Complex Valued Nonlinear Adaptive Filters: Noncircularity, Widely Linear and Neural Models, John Wiley \& Sons, 2009.

[15] T. Y. Al-Naffouri and A. H. Sayed, "Transient analysis of datanormalized adaptive filters," IEEE Trans. Signal Process., vol. 51, no. 3, pp. 639-652, 2003. 